\title{
Amiodarone-Induced Thyrotoxicosis in a Patient With Post- Ablative Hypothyroidism Controlled for 50 Years Presenting as Psychosis
}

\author{
Teresa Ratajczak $^{\mathrm{a}, \mathrm{c}}$, Kristy Zechiel ${ }^{\mathrm{b}}$, Irina Overman ${ }^{\mathrm{a}, \mathrm{b}}$, Ankur Gupta $^{\mathrm{a}}$
}

\begin{abstract}
Thyroid gland abnormalities and atrial fibrillation are common medical conditions that occur in the geriatric patient. We present a case of an 85-year-old female with a remote history of radioiodine ablation of the thyroid, on levothyroxine therapy who developed amiodaroneinduced thyrotoxicosis. Patient was initiated on amiodarone for history of chronic atrial fibrillation 5 months prior to admission. She presented with acute psychosis in the form of visual hallucinations due to thyrotoxicity. The goal of this article is to review the effects of amiodarone on the thyroid gland and emphasize presentation of hyperthyroidism in an elderly patient.
\end{abstract}

Keywords: Hyperthyroidism; Amiodarone; Thyrotoxicosis

\section{Introduction}

Amiodarone is a commonly used antiarrhythmic medication. Three percent of patients treated with it develop thyroid dysfunction in the form of amiodarone-induced thyrotoxicosis (AIT) [1]. We report a case of an 85-year-old female with a remote history of radioiodine ablation of the thyroid, treated with levothyroxine along with amiodarone for chronic atrial fibrillation, who presented with weakness of 5 days and visual hallucinations of 1-month duration.

\section{Case Report}

An 85-year-old female presented from home to the emergency department with weakness and visual hallucinations. The weakness was described as a generalized sense of heaviness

\footnotetext{
Manuscript accepted for publication September 22, 2015

aDepartment of Internal Medicine, Wright State University, Dayton, OH, USA ${ }^{b}$ Department of Geriatrics, Wright State University, Dayton, OH, USA

${ }^{\mathrm{c} C o r r e s p o n d i n g ~ A u t h o r: ~ T e r e s a ~ R a t a j c z a k, ~ D e p a r t m e n t ~ o f ~ I n t e r n a l ~ M e d i c i n e, ~}$ Wright State University, Dayton, OH, USA.

Email: Teresa.ratajczak@wright.edu
}

doi: http://dx.doi.org/10.14740/jmc2317w that has been present for the past 5 days, impairing ambulation. She reported seeing objects in her house such as trees, roads and construction supplies. The hallucinations had been occurring for 1 month; however, they were not distressing to the patient, as she was aware that the images were not real. She denied any auditory hallucinations. New medications included quetiapine, for the visual hallucinations, initiated 3 weeks earlier by her primary care physician. The patient also reported functional decline over 6 months, requiring assistance with medications, finances, shopping and transportation.

Her medical history was significant for acquired hypothyroidism due to Graves' disease treated with radioiodine ablation in her twenties, hypertension, esophageal reflux disease and atrial fibrillation. Patient's arrhythmia was controlled with sotalol for 4.5 years. The treatment was changed to amiodarone 5 months prior to admission as an elective cardioversion was planned. After 3 months of amiodarone therapy, cardioversion was performed, which temporarily restored sinus rhythm. Unfortunately, due to recurrent atrial fibrillation, amiodarone was continued. The following week, the patient developed symptoms of lower extremity edema and weakness. Amiodarone was discontinued and diltiazem and digoxin were started. Other home medications consisted of quetiapine, rabeprazole, furosemide, warfarin and levothyroxine. Review of medical records showed that the patient's thyroid function had always been controlled with levothyroxine dosing of $25-50$ $\mu \mathrm{g}$ daily.

Physical exam was significant for tachycardia, irregularly irregular rhythm and trace lower extremity edema. No thyromegaly, lid lag, or exophthalmos were noted. Musculoskeletal exam revealed preserved upper and lower extremity muscle tone. The patient's vision, checked with a Snellen chart, was noted to be 20/70 bilaterally. Complete blood count, urinalysis, troponin, B12, folate and renal panel were unremarkable. Digoxin level and INR were in the therapeutic range. CT scan of the head and chest X-ray did not show any intracranial or cardiopulmonary pathology. ECG showed atrial fibrillation.

The low dose of thyroid hormone replacement prompted consideration of a thyroid gland dysfunction. Admission TSH testing showed results of $<0.005 \mu \mathrm{IU} / \mathrm{mL}$ (reference range: $0.40-4.00 \mu \mathrm{IU} / \mathrm{mL}$ ) with prior reading of $1.8 \mu \mathrm{IU} / \mathrm{mL} 5$ months earlier (Table 1). Free T4 was 3.44 (reference range: $0.80-1.80 \mathrm{ng} / \mathrm{dL})$ and free T3 was $5.4(2.30-4.20 \mathrm{pg} / \mathrm{dL})$. TSI level was $<24$ (reference range: $<140 \mathrm{U} / \mathrm{mL}$ ). 
Table 1. Patient's Thyroid Studies Prior to and During Amiodarone Treatment

\begin{tabular}{lllll}
\hline Date & $\begin{array}{l}\text { TSH }(\boldsymbol{\mu I U} / \mathbf{m L}) \\
\text { (reference range } \\
\mathbf{0 . 4 0 - 4 . 0 0 )}\end{array}$ & $\begin{array}{l}\text { Free T4 }(\mathbf{n g} / \mathbf{d L}) \\
\text { (reference range: } \\
\mathbf{0 . 8 0}-\mathbf{1 . 8 0})\end{array}$ & $\begin{array}{l}\text { Free T3 }(\mathbf{p g} / \mathbf{m L}) \\
\text { (reference range: } \\
\mathbf{2 . 3 0}-\mathbf{4 . 2 0})\end{array}$ & Antiarrhythmic agent \\
\hline May 2012 & 1.0 & 0.94 & & sotalol \\
November 2013 & 0.56 & 1.20 & sotalol \\
\hline May 2014 & 1.8 & 1.03 & 5.4 & sotalol/amiodarone started May 26, 2014 \\
\hline October 2014 & $<0.005$ & 3.44 & methimazole started 10 mg \\
\hline January 06, 2015 & 19.1 & 0.52 & methimazole changed to 5 mg \\
\hline January 29, 2015 & 0.030 & 2.35 & methimazole discontinued \\
\hline March 24, 2015 & 2.7 & 0.81 & \\
\hline
\end{tabular}

*Amiodarone discontinued September 29, 2014.

Thyroid doppler ultrasound revealed an enlarged left lobe of the thyroid, relatively hypervascular, without a discrete mass and an atrophic right lobe. A nuclear medicine thyroid uptake scan showed minimal uptake of technetium 99m/iodine-131 in the left thyroid lobe. No significant activity was seen in the right thyroid lobe. The 24-h tracer uptake was $1 \%$ (normal range $9-33 \%$ ). It was felt that the patient's presentation was consistent with amiodarone induced thyrotoxicosis, likely due to residual thyroid activity. She was started on methimazole $10 \mathrm{mg}$ and levothyroxine was discontinued. Her weakness and visual hallucinations improved during the course of inpatient stay. Several months after discharge, the patient reported improvement in both her mental and physical function during a primary care follow-up visit. The methimazole dosing was decreased to $5 \mathrm{mg} 3$ months after treatment started, and then discontinued a month later due to euthyroid hormone labs.

\section{Discussion}

Amiodarone is an iodine-rich class III antiarrhythmic agent that induces thyrotoxicosis in approximately $3 \%$ of patients in North America [1]. It resembles thyroxine in its molecular structure, and contains about $75 \mathrm{mg}$ of iodine in each $200 \mathrm{mg}$ tablet [1,2]. This leads to release of approximately $8 \mathrm{mg}$ of free iodine into the circulation. The medication is very lipophilic, with a long half-life, accumulating in tissues as well as the thyroid gland [1,2]. For comparison, the recommended daily dose of iodine is $150 \mu \mathrm{g}$ for adults over 18 years of age. Therefore, standard daily dosing of $200 \mathrm{mg}$ amiodarone provides approximately 20 - 40 times the daily iodine requirement.

There are two main forms of amiodarone induced thyrotoxicosis (AIT). Type 1 AIT is an iodine-induced hyperthyroidism, also known as the Jod-Basedow effect, which occurs in patients with an underlying abnormal autonomous thyroid tissue. The iodine load causes the gland to function independently without the negative feedback control of the pituitary gland $[3,4]$. Type 2 AIT occurs in patients with a previously normal thyroid gland. The extra iodine causes a destructive thyroiditis, which leads to release of preformed, stored hormones from the damaged thyroid follicular cells [3, 4]. Diagnosis of thyrotoxicosis is based on detection of increased free thyroid hormone concentrations and suppressed thyroid stimulating hormone TSH levels in the serum. Imaging consists of performing an ultrasound of the gland which will show increase in gland size, with a hypoechoic or nodular pattern in type 1 thyrotoxicosis, and a normal gland size in type 2 [5]. Another modality used is nuclear imaging with thyroid radioactive iodine (RAI) uptake, however this technique is not very useful in iodine replete areas. This is because high levels of iodine in the serum compete with the tracer, showing a low or low-normal uptake in both types [3].

Type 1 AIT is treated with either methimazole or propylthiouracil. These medications work by blocking the synthesis of thyroid hormones [6]. They are usually continued for $6-18$ months and are stopped when the urine iodine levels return to normal. Additional beta-blockade may be necessary, as stopping amiodarone may further exacerbate symptoms of thyrotoxicosis, due to the fact that the medication also inhibits peripheral T4 to T3 conversion [6]. Patients with type 2 AIT are treated by discontinuation of amiodarone. If the thyrotoxicosis is mild with no cardiac symptoms, amiodarone can be continued with the addition of steroids. Prednisolone $40-60 \mathrm{mg}$ /day is recommended for one to three months with a slow taper [6].

Radioactive iodine has been used as a treatment for hyperthyroidism since the 1940s and treatment regimens over the years have varied from high or low doses of radioiodine [7]. Historically, the initial goal was to destroy the thyroid tissue and establish an euthyroid state. Although this treatment was highly effective, it led to hypothyroidism in $70-80 \%$ of the patients [7]. Clinicians adapted to administering a lower dose of iodine, with reduced incidence of hypothyroidism; however, the cure rates were unacceptably low. Later studies demonstrated that development of hypothyroidism is inevitable, and independent of the amount of radioiodine administered, with an annual progression of 2-3\% [8]. The current goal of treatment is to establish a hypothyroid state, usually within $3-6$ months. The recurrence of hyperthyroidism is thought to be less than 3\%. Late recurrence of the disease, especially decades after treatment is rare, with only a few cases reported in literature [9].

This case highlights the importance of recognition of the signs and symptoms of hyperthyroidism in the elderly patients, as they may not present with the classic symptoms seen 
in younger patients. Diaphoresis, heat intolerance, tremor and nervousness are typically absent. The three signs more frequently present in thyrotoxic elderly patients are apathy, tachycardia and weight loss [10]. The term apathetic hyperthyroidism refers to a flat affect, lethargy and depression. Conversely, cardiac arrhythmias such as atrial fibrillation are more frequent in the elderly. Other presenting symptoms include agitation, confusion, decreased activity, fatigue or weakness [11]. These are often misinterpreted as being related to aging or other illnesses [10]. New onset psychosis is an uncommon presentation $(<1 \%)$ of hyperthyroidism that also needs to be excluded in all patients [12].

Our patient presented with 1 month of visual hallucinations, consistent with psychosis as a most apparent symptom of recurrent Graves' disease. We believe that her diagnosis is consistent with type 1 AIT due to prior history of Graves with autonomous thyroid activity. Although treated with radioactive iodine in the past, it is likely this did not achieve a complete ablation of her thyroid gland. What is interesting, with only a few such cases reported in literature, is the relapse of her Graves' disease many years after radioiodine therapy. The low daily dose of her thyroid hormone replacement lead the team to consider recurrence of Graves' disease. The patient was able to return to her prior level of function with the proper treatment of her condition.

\section{Conclusion}

The unique features of this case are the presentation of thyrotoxicosis as psychosis, many decades after radioiodine treatment. This case highlights the importance of excluding secondary organic causes in a previously stable elderly patient with a sudden clinical change. This should also be investigated, especially in patients with a history of amiodarone therapy.

\section{References}

1. Tsang W, Houlden RL. Amiodarone-induced thyrotoxicosis: a review. Can J Cardiol. 2009;25(7):421-424.

2. Goodman \& Gilman's The Pharmacological Basis of Therapeutics, 11th Edition.

3. Martino E, Bartalena L, Bogazzi F, Braverman LE. The effects of amiodarone on the thyroid. Endocr Rev. 2001;22(2):240-254.

4. Markou K, Georgopoulos N, Kyriazopoulou V, Vagenakis AG. Iodine-Induced hypothyroidism. Thyroid. 2001;11(5):501-510.

5. Daniels GH. Amiodarone-induced thyrotoxicosis. J Clin Endocrinol Metab. 2001;86(1):3-8.

6. Osman F, Franklyn JA, Sheppard MC, Gammage MD. Successful treatment of amiodarone-induced thyrotoxicosis. Circulation. 2002;105(11):1275-1277.

7. Becker DV, Sawin CT. Radioiodine and thyroid disease: the beginning. Semin Nucl Med. 1996;26(3):155-164.

8. Franklyn JA, Daykin J, Drolc Z, Farmer M, Sheppard MC. Long-term follow-up of treatment of thyrotoxicosis by three different methods. Clin Endocrinol (Oxf). 1991;34(1):71-76.

9. Hegele RA, Volpe R. Relapse of Graves' disease 23 years after treatment with radioactive iodine (131I). J Clin Lab Immunol. 1985;18(2):103-105.

10. Boelaert K, Torlinska B, Holder RL, Franklyn JA. Older subjects with hyperthyroidism present with a paucity of symptoms and signs: a large cross-sectional study. J Clin Endocrinol Metab. 2010;95(6):2715-2726.

11. Arnold BM, Casal G, Higgins HP. Apathetic thyrotoxicosis. Can Med Assoc J. 1974;111(9):957-958.

12. Gagliardi JP, Clary GL. Treatment of Thyrotoxicosis-Induced Psychosis. Psychopharmacol Bull. 2002;36(4):713 . 